\title{
Near-field Acoustical Holography without the Errors and Limitations Caused by the Use of Spatial DFT
}

\author{
Rolf Steiner \\ McKinsey\&Comp, Alpenstrasse 3, 8065 Zurich, Switzerland \\ JHrgen Hald \\ BrLel \& Kj6r, S\&V Measurement A/S, Skodsborgvej 307, 2850 N6rum, Denmark
}

(Received 15 May 1999; accepted 15 September 2000)

\begin{abstract}
The present paper describes a Statistically Optimal Near-field Acoustical Holography (SONAH) method, which avoids the errors caused by the use of spatial DFT/FFT in conventional planar Near-field Acoustical Holography (NAH). The SONAH method performs a statistically optimal sound field reconstruction, the plane-to-plane calculations being performed in the spatial domain instead of the spatial frequency domain. Furthermore the method allows an arbitrary positioning of the measurement points in the hologram plane. This allows for optimisation of the microphone positions, for example the use of a sparse measurement grid. For a small $6 \times 6$ array, it is shown by means of simulations that the new algorithm performs better than the DFT-based NAH method.
\end{abstract}

\section{INTRODUCTION}

The plane to plane propagation of the sound field away from the source can be described mathematically to be a $2 \mathrm{D}$ spatial convolution with a propagation kernel. It is well known that in the Fourier domain this convolution reduces to a simple multiplication by a transfer function. This is equivalent to the Fourier transform of the propagation kernel. In NAH the Fourier transform is implemented as a spatial DFT/FFT of the pressure data measured over a finite area. A requirement introduced by the $2 \mathrm{D}$ DFT processing is the need for a regular rectangular measurement grid.

The use of spatial DFT/FFT and multiplication with a transfer function in the spatial frequency domain is computationally very efficient. It does, however, introduce some errors. The implicit introduction of a spatial window causes spectral leakage in the spatial frequency domain. This shows up as "window effects" in the calculation plane. The discrete representation in the spatial frequency domain introduces periodic replica in the spatial domain. This causes "wraparound errors" in the calculation plane. ${ }^{1}$ Various techniques have been introduced to reduce these errors. See for example references ${ }^{2,3}$. To the authors' knowledge, however, no holographic reconstruction algorithm, which is optimal in a broad sense, and which avoids the use of spatial DFT processing, has been presented yet. The new SONAH method performs the plane-to-plane transformation directly in the spatial domain rather than via the spatial frequency domain.

\section{THE MODEL}

We shall consider measurement set-ups of the type illustrated in Fig. 1. In this figure a rectangular $(x, y, z)$ coordinate system has been introduced and the following assumptions have been made. The half space $z \geq z_{S}$ to the right of the source plane, $z=z_{S}$, is source free and with a homoge- neous medium (air). In this half space the sound field satisfies the homogeneous wave equation. A set of microphones measures the sound pressure $p(\mathbf{r})$, which is assumed to have harmonic time dependence. The position vector is $\mathbf{r} \equiv[x, y, z]^{\mathrm{T}}$, and the implicit time dependence is given by $e^{j \omega t}$, where $\omega$ is the angular frequency. All the microphones lie in the hologram plane $z=z_{H}>z_{S}$, but apart from that they can be freely positioned. For example they are not restricted to a regular rectangular grid. The hologram plane is parallel to the source plane.

With reference to Fig. 1, we seek a relation between the sound pressure $x(\cdot)$ in the source plane $z=z_{S}$ (the source sig$n a l)$ and the sound pressure $y(\cdot)$ in the hologram plane $z=z_{H}$ (the hologram signal). We shall later look at the reconstruction of the sound field in other parallel planes $z=z_{R}>z_{S}$. However, the source plane is of special interest, since among the planes parallel to the hologram plane, it is the one nearest to the sources and it is located so that the volume between it and the hologram plane is source free. Both of these signals are continuous functions of the two spatial co-ordinates $x$ and $y$, which define the two-dimensional reduced position vector $\overline{\mathbf{r}} \equiv[x, y]^{\mathrm{T}}$. The source signal $x(\overline{\mathbf{r}})$ produces a sound field that propagates into the right half space $z>z_{S}$ and subsequently stimulates the signal $y(\overline{\mathbf{r}})$ in the hologram plane. This mapping of the source signal $x(\cdot)$ into the hologram signal $y(\cdot)$ shall be called the acoustical mapping and it is represented by the symbol $g: y(\overline{\mathbf{r}})=(g x)(\overline{\mathbf{r}})$. In the spatial angular frequency domain (the wave number domain), $\overline{\mathbf{k}} \equiv\left[k_{x}, k_{y}\right]^{\mathrm{T}}$, the acoustical mapping is a simple multiplication: ${ }^{1}$

$$
\begin{aligned}
& Y(\overline{\mathbf{k}})=G\left(\overline{\mathbf{k}}, z_{H}-z_{S}\right) X(\overline{\mathbf{k}}) \\
& G(\overline{\mathbf{k}}, \Delta z) \equiv e^{-j k_{z} \Delta z} \\
& k_{z} \equiv \sqrt{k^{2}-k_{x}^{2}-k_{y}^{2} ;} \\
& k \equiv \omega / c
\end{aligned}
$$

